\title{
Physical Activity Intervention for Loneliness (PAIL) in community-dwelling older adults: protocol for a feasibility study
}

\author{
Anastasia V. Shvedko ${ }^{1 *}$ D, Janice L. Thompson', Carolyn A. Greig ${ }^{1,2}$ and Anna C. Whittaker
}

\begin{abstract}
Background: Low-quality social relationships in older adults are strongly associated with feelings of loneliness. Physical activity interventions could reduce loneliness and improve psychological well-being, among other health benefits. The aim of this study is to examine the feasibility of a Physical Activity Intervention for Loneliness (PAIL) in community-dwelling older adults at risk for loneliness.

Methods/design: This feasibility study is a two-arm randomised controlled trial (RCT) with a wait-list control group using a mixed-methods research design. The primary aim of the feasibility study is to estimate recruitment, retention and adherence rates; the appropriateness of the intervention design and its practicality; the acceptability of the intervention by participants; and the set of instruments and measures and primary outcome measures to inform a future large-scale randomised trial. After eligibility screening, randomisation will be conducted using computer-based random sequence generation. Baseline and post-intervention assessments for intervention and control groups will include height, weight, body mass index, resting blood pressure, physical activity using accelerometry, loneliness, social support, social networks, anxiety and depression, self-efficacy for exercise, satisfaction with social contacts, and expected outcomes and barriers for exercise using questionnaires. Focus groups will be conducted at the mid-point and post-intervention period using a phenomenological approach to analyse the participants' experiences of taking part in PAlL.
\end{abstract}

Discussion: This trial will provide important information regarding the feasibility of PAIL in community-dwelling older adults at risk for loneliness using a mixed-methods approach combining quantitative and qualitative research methods.

Trial registration: Clinicaltrials.gov NCT03458793

Keywords: Feasibility study, Physical activity, Loneliness, Older adults, Randomised controlled trial

\section{Background}

Maintenance of social connectedness throughout the lifespan is an important aspect of successful ageing [1]. The disruption of established social patterns or poor quality of social relationships negatively impacts quality of life and well-being in older adults and is highly associated with loneliness $[2,3]$. According to UK statistics, loneliness is highly prevalent and increasing among older adults aged 65 years or older [4]. Further, $11 \%$ of

\footnotetext{
* Correspondence: axs1235@bham.ac.uk

'School of Sport, Exercise and Rehabilitation Sciences, University of Birmingham, Birmingham, UK

Full list of author information is available at the end of the article
}

people aged 75 years and older reported to have no close friends and visited their general practitioner (GP) to fulfil their need to talk to somebody [4]. Defined as a discrepancy between a person's desired and actual social relationships [5], loneliness and a lack of social relations were considered to be high-risk factors for morbidity and mortality, and the negative impact of loneliness can be as harmful as smoking 15 cigarettes a day [6,7]. Bearing in mind the ageing of the UK population, with a sharp increase in the proportion of adults aged 65 years and older over the last 30 years [8], health professionals have placed special emphasis on the promotion of "active ageing" enabling older adults to increase 
participation in "social, economic, cultural, spiritual and civic affairs" to maintain quality of life [9]. Further, promotion of a variety of campaigns to prevent loneliness [10-12] may reduce or slow the burden on NHS expenses, with potential economic benefits estimated at around $£ 900$ per annum per person associated with loneliness reduction [10]. Given this, the early prevention of loneliness and timely implementation of health interventions in a community setting to tackle the problem at its early onset seems prudent.

Physical activity (PA) is defined as any bodily movement produced by the contraction of skeletal muscles that results in a substantial increase over resting energy expenditure $[13,14]$. It is a health behaviour that can facilitate meaningful social relationships and serve as an alternative to medical treatments which may have negative side effects $[11,15,16]$. Compared to other forms of treatment therapies (e.g. mindfulness therapy, art and craft therapy), physical activity interventions, especially in small groups (up to eight to nine people), can assist in building friendly and trusted relationships between participants based on shared interests and similar needs, as demonstrated by previous research [12, 17].

Mechanisms of physical activity interventions' effectiveness are suggested to relate to loneliness reduction models, stress reduction and increased social support during activities. Related to the first mechanism, the social compensation model [18] suggests that PA can work via compensation for lost meaningful social connections due to increased peripheral social networking during friendly conversations between participants [17]. The hypothesis of the broaden-and-build theory of positive emotions [19] posits that enjoyable forms of PA generate happiness and bring positive emotions, which in turn could be associated with loneliness reduction as shown in a longitudinal study Newall et al. [20]. The tripartite model of group identification was found to be effective, particularly among lonely seniors, based on the sense of identification and social attraction to group members with shared interests and goals, arising during engagement in physical activities [21]. Related to the second mechanism, based on the stress/social support model [22], social networks promote well-being that is associated with loneliness reduction in older adults. Among the mechanisms named above, only the tripartite model of group identification was shown to be successfully applied in the treatment of loneliness directly in the context of PA.

Based on the analysis of existing randomised controlled trials (RCTs), there are few PA interventions for loneliness reduction conducted with residents in community settings [23]. This is also in line with previous systematic reviews and meta-analyses [2, 15, 24-30]. A subsequent analysis for other loneliness-related social outcomes has shown that for social functioning (as a sub-domain of health-related quality of life), specific aspects of PA interventions can successfully influence social health [23] with the strongest effects being obtained for group setting exercise interventions, with delivery by a health/medical professional, in a diseased rather than healthy population. PA interventions did not appear to be effective for increasing social support or social networks [23]. In addition, the majority of studies used a cross-sectional design, which does not allow determination of causality and limits the rigour of the research evidence. However, longitudinal studies do not always support the direct effects of PA interventions on loneliness reduction in older adults [15]. Nevertheless, it is important to emphasise that the majority of existing PA interventions assess loneliness as a secondary outcome within a number of other psychosocial outcomes, which limits the ability to fully examine these interventions' effectiveness for reducing loneliness [15, 31].

Further, a number of moderating (affecting the strength of the relationship between PA and loneliness) and mediating factors (driving the influence of PA on loneliness) are not consistently considered in intervention studies, making the outcomes of even well-conducted interventions less credible [15]. Research shows that global- [32, 33] and domain-specific self-efficacy [34] are moderating factors, and social support was found to be both a moderating [35] and mediating factor [36]. Also, perceived self-efficacy in one study by Fry and Debats [34] was found to be a superior predictor of loneliness and psychological distress in older adults compared to demographic factors, physical health and support networks. Further, personal (self-efficacy) and environmental (social support) variables moderating relationships between loneliness and PA have a bidirectional link with each other [37]. Perceived social support is a moderator of self-efficacy, and increased self-efficacy leads to better social support [35].

Bearing in mind the limitations of the current literature as presented above, understanding the mechanisms of association between loneliness and PA may bring new insights to the designing of novel and effective PA interventions [15]. Further research is needed to explore the association between loneliness, self-efficacy and social support in the context of PA interventions for older adults. However, before the mechanisms can be fully understood, the practicalities and feasibility of implementation of such interventions with older adults must be tested. The aim of the study is an examination of the feasibility of RCT of Physical Activity Intervention for Loneliness (PAIL) in community-dwelling older adults at risk for loneliness. For the planned future large-scale RCT, the primary hypothesis is that, compared with the inactive control group, participants in 
the intervention group will report a greater decrease in loneliness. The secondary hypothesis is that participants in the intervention group will significantly increase their amount of physical activity engagement per week, and this will be associated with greater positive changes in other psychosocial and health outcomes compared to control group participants.

\section{Study objectives}

The following specific aims of this feasibility study are to estimate:

1. Recruitment rate, attendance and retention rates (number of participants completing the study as a proportion of those randomised)

2. The appropriateness and practicality of the designed intervention in the proposed settings

3. The acceptability of the intervention by participants and willingness to participate

4. The appropriateness of the assessment tools

5. The appropriateness of the statistical methods of data analysis used

6. The power calculation of the likely required sample size for a future large-scale RCT.

7. The acceptability of measures and the most suitable primary outcome measure for a future large-scale RCT

In addition, to reflect the aims of a future large-scale RCT that this feasibility study seeks to inform, the effect sizes between the intervention and control groups will be examined.

\section{Methods/design}

PAIL is a tw0-arm RCT of a 12-week intervention compared with a wait-list control (WLC) condition using a mixed-methods research design (including quantitative and qualitative research methods). Quantitative data will be collected from the intervention and control groups at the baseline and immediate post-intervention periods. Qualitative data aimed to assess the appropriateness, practicality and acceptability of the designed intervention in the proposed settings will be collected at the mid-point and post-intervention period. The trial was approved by the Science, Technology, Engineering and Mathematics (STEM) Research Ethics Committee of the University of Birmingham, UK (ERN_16-1419A). Consent forms (Additional file 1) will be obtained from all participants prior to entry into the study. This protocol for a feasibility study was guided by the SPIRIT 2013 Checklist (Additional file 2).

\section{Eligibility}

Participants will be recruited from local neighbourhoods (households) and community centres across the wide geographic area of Birmingham, UK.

\section{Inclusion criteria}

The following are the inclusion criteria:

1. Community-dwelling older adults aged 60 years and older;

2. Previously sedentary (i.e. as defined by a lack of regular involvement in more than 20 min exercise per week over the past month that increased breathing significantly and was considered moderate) [38];

3. At risk of loneliness and having $\geq 6$ out of 9 points on the three-item loneliness scale during the phone screening [39] (Additional file 3);

4. Physically mobile as measured using the Short Physical Performance Battery (SPPB) [40] with a score $\geq 9$ out of 12 [41];

5. Healthy or having one or more common chronic diseases but ambulatory, without a cognitive disability as assessed by the Montreal Cognitive Assessment (MOCA) [42] with a score $\geq 22$ out of 30 [43];

6. Able to give written informed consent;

7. English speaking and able to complete paper and pencil questionnaires.

\section{Exclusion criteria}

The following are the exclusion criteria:

1. < 60 years old;

2. Currently taking part in another physical activity intervention;

3. Socially active or not lonely based on the phone screening tool by Hughes et al. [39];

4. Regularly physically active;

5. Moderate to severe cognitive disability with cut-off below 22 for MOCA or clinical diagnosis of dementia or Alzheimer's disease;

6. Not ambulatory, i.e. not able to walk $4 \mathrm{~m}$;

7. Not literate in English (speaking and reading) as this precludes taking pen and paper tests.

\section{Interventions}

The PAIL feasibility study is a 12-week intervention consisting of group walking and health educational/social interaction workshops performed once weekly for a duration of up to $90 \mathrm{~min}$ per session. The design and features of the PAIL intervention are based on the features of effective interventions that were obtained from a systematic review and meta-analysis of the existing evidence conducted by Shvedko et al. [23]. Effective interventions were physical activity (PA) rather than PA interventions with social interactions, organised in group settings versus individually or one-to-one, delivered by a medical healthcare provider versus nonqualified providers and with strongest effects obtained for diseased versus healthy populations. 
Group walking sessions will be run once weekly for up to $45 \mathrm{~min}$ each in small groups (up to eight to nine people per group) and delivered by a trained walk leader (i.e. level 3 certified personal trainer and a group exercise instructor). Prior to the first walking session, participants in the intervention group will be asked to complete the "Par-Q and You form" [44] and will receive a copy of general practitioner (GP) letter that informs their GP about their participation in the study. Participants attending the walking intervention will join the walking leader following a specified route in varied locations at every session to maintain interest in the intervention [45]. During guided walking, the instructor will be acting as a facilitator of social contact by using in-session talks and friendly discussion between participants to reduce psychosocial tension. Walking sessions will be based on the principles of gradual progression and adaptation to PA [13]. The intensity of the walks will be monitored objectively by heart rate monitors using the age-predicted heart rate maximum $\left(\mathrm{HR}_{\max }\right)$ method [46] by calculating in advance the age-predicted zones of heart rate intensity using the formula 220 minus age in years. Additionally, the intensity of walks will be monitored subjectively using the $0-10$ Borg Ratings of Perceived Exertion (RPE) Scale [47] and the talk test [48]. Participants will be provided with a standardised set of instructions about the use of the 0-10 Borg RPE Scale $[47,49]$ and talk test $[48]$ to enhance their understanding of these methods before the intervention [13]. Light-to-moderate intensity walking will be monitored by the ability of participants to talk back comfortably during exercises using the talk test [48] and 2-4 on the 0-10 Borg RPE scale [47]. A warm-up will be performed at the beginning of each session for 7-10 min and will include preparatory dynamic and static stretches standing, performed for the major muscle groups and walking at a leisurely pace maintaining an upright pose. At the end of the walk, participants will perform up to 10 min of balance training recommended for older adults to reduce frailty [50]. A further cool down will be performed at the end of the walking session for 5-7 min and will consist of stretching exercises for upper and lower body performed in two regimes: maintenance (e.g. maintenance of the muscle length) and developmental stretches (e.g. involving the gradual lengthening of a muscle group into an elongated position and subsequent "hold" of this position). At the end of the cool-down, participants will perform breathing exercises while standing for about 2 min.

Group walking sessions will be followed by the health education/social interactions workshops delivered in the form of a group presentation weekly for up to 45 min by the researcher ( $\mathrm{PhD}$ student) on a variety of healthy ageing topics such as eye hygiene, mental health and well-being, preventing falls, social support, nutritional guidelines and physical activity recommendations for older adults (Additional file 4).

\section{Intervention group}

After randomisation, participants in the intervention group will start the 12-week walking intervention.

\section{WLC group}

Participants in the wait-list control (delayed intervention) group will start the intervention after their follow-up measures are completed approximately 12 weeks post-randomisation.

\section{Measures}

All measures will be conducted at the host academic institution at baseline and at the immediate post-intervention period (Fig. 1) except for focus groups, which will take place at the mid-point and post-intervention.

\section{Socio-demographic characteristics and medical conditions}

Participants will provide socio-demographic information including their age, gender, ethnicity, marital status, living arrangements, level of education, any children, employment status and any medical conditions.

\section{Health measures}

Cognitive function will be assessed using a Montreal Cognitive Assessment (MOCA) scale designed to test mild cognitive impairment [42]. Physical functioning will be assessed using the Short Physical Performance Battery (SPPB) [40]. Height to the nearest $0.1 \mathrm{~cm}$ will be measured using a stadiometer (Seca AG, Reinach, Switzerland) and recorded in metres. Weight will be assessed using weighing scales (Tanita UK Ltd., Middlesex, UK) to the nearest $0.1 \mathrm{~kg}$. Body mass index (BMI, $\mathrm{kg} \mathrm{m}^{-2}$ ) will be calculated by dividing mass $(\mathrm{kg})$ by height squared $\left(\mathrm{m}^{2}\right)$. Resting blood pressure (BP rest, $\mathrm{mmHg}$ ) will be measured using a portable semi-automatic OMRON sphygmomanometer (OMRON HEM705CP sphygmomanometer; Omron Matsusaka Co Ltd., Japan). Physical activity will be measured using ActivPAL accelerometers (PAL Technologies Ltd. Glasgow, UK) at baseline and immediate postintervention period over a continuous 7-day period of awake and sleeping ( $24 \mathrm{~h}$ a day) except when bathing or swimming [51].

\section{Questionnaires}

Loneliness will be assessed using the 8-item UCLA Loneliness Scale (UCLA-8) [52]. Social support will be assessed using the 20-item Medical Outcomes Study Social Support Survey (MOSSSS) [53]. Social networks will be assessed using the 6-item Lubben's Social 


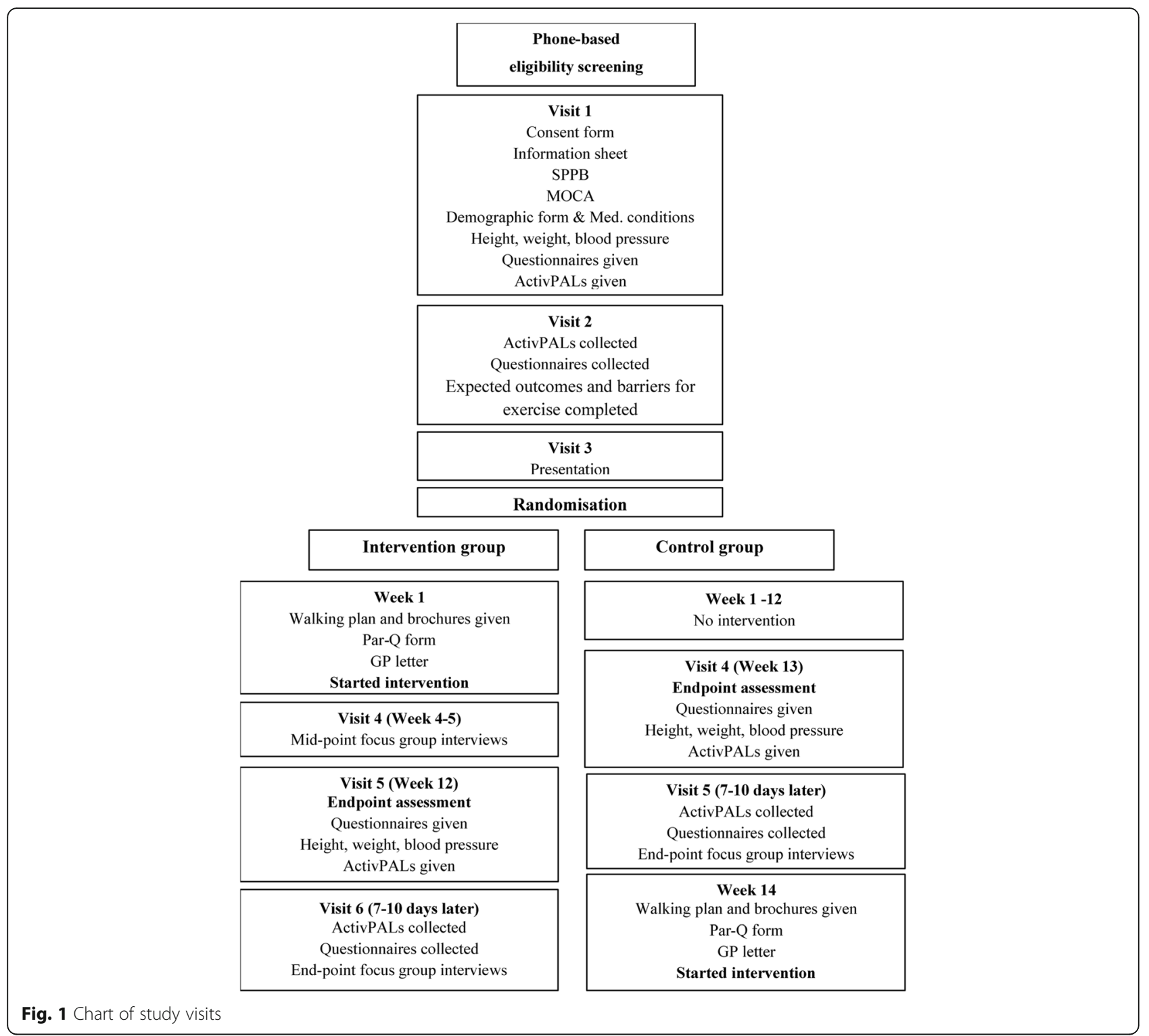

Network Scale (LSNS-6) [54]. Depression and anxiety will be assessed using the 14-item Hospital Anxiety and Depression Scale (HADS) [55]. Self-efficacy for exercise will be measured using the revised 9-item Self-Efficacy for Walking/Exercise Scale (SEE) using a paper-andpencil format [56]. Satisfaction with level of social contacts will be measured with the question "How satisfied are you with your social contacts?" [57]. Expected outcomes and barriers for exercise will be administered using the Expected Outcomes and Barriers for Habitual Exercise scale [58] adapted for the older adult population. Four questions related to sport competence have been deleted from the expected outcomes sub-scale due to irrelevance for this population group [58]. The expected outcomes and barriers for exercise scale have demonstrated good internal consistency from
0.66 to 0.85 and a high test-retest reliability of 0.78 in previous research [58].

\section{Qualitative assessments}

To understand participants' experiences of taking part in the PAIL feasibility trial, focus groups will be conducted at mid-point (between the weeks 4 and 5) and at the end of the 12-week intervention using semi-structured discussions in small groups (four to nine people per group) of mixed gender (Additional files 5 and 6). The research team will check if any alterations will be required based on the participants' feedback. The 32-item checklist for reporting qualitative research will guide the researcher to ensure clarity and transparency in focus groups methodology [59]. Focus groups will be audio recorded using a digital recorder and will be transcribed verbatim. An 
independent trained focus group leader will act as a moderator and facilitator of the focus groups [60].

\section{Feasibility outcomes}

The following feasibility outcomes will be assessed in this study:

1. Attendance will be calculated as the total number of attended sessions divided by the total number of sessions of the intervention and recorded as a percentage;

2. Recruitment rate will be calculated as the number of individuals responding to advertisements and friends' referrals out of a total number of formal invitations given/advertisements placed (including web-based advertisements, advertisements placed in the local cohort groups and poster and leaflet material disseminated in the community). Recruitment rate will be recorded as a percentage, e.g. $25 \%$ (48/195). It is acknowledged that advertisements may reach a large number of individuals but it is impossible to quantify this;

3. Retention rate will be calculated as the number of participants completing the study as a proportion of those randomised;

4. The appropriateness, practicality and acceptability of the designed intervention in the proposed settings will be assessed using focus group interviews. The focus group transcripts will be analysed using a phenomenological inductive approach [61], and these data will be used to help the research team to improve the quality of the delivered intervention by informing positive changes in the methodology and design of the intervention for the future implementation in a consequent study;

5. The assessment rate of questionnaires will be evaluated as the total number of completed questionnaires divided by the total number of questionnaires and recorded as a percentage;

6. The appropriateness of statistical methods of data analysis will be analysed by the research team in terms of suitability to the data;

7. A power calculation and sample size estimation will be calculated for meaningful potential future primary outcomes (e.g. loneliness or social support) using a method based on the differences in means between the intervention and control groups, using the Gpower software [62];

8. The effect size (ES) will be calculated for loneliness, social support, social networks, anxiety and depression, self-efficacy for exercise, satisfaction with level of social contacts and the expected outcomes and barriers for exercise. Means (M) and standard deviations (SD) will be used to investigate the effect size for change in loneliness using mixed between (intervention group vs. control group) and within (over time) repeated-measures analysis of variance (ANOVAs) with post-hoc comparisons.

\section{Data monitoring}

The data monitoring committee for this project is the supervisory research team (three academic supervisors) who are independent of the trial sponsor. They will be responsible for checking data accuracy upon assembly of the final database following completion of data collection prior to data analysis. The $\mathrm{PhD}$ student is responsible for monitoring and reporting spontaneous adverse events or any unintended trial effects to the supervisory team, and the PI (Whittaker) is responsible for reporting these to the sponsor. The trial is also subject to independent audit request by the sponsor, the University of Birmingham, by a team independent to the supervisory/research team. The sponsor contact details are given below.

Dr Sean Jennings, Head of Research Governance, University of Birmingham (e-mail: s.jennings@bham.ac.uk).

\section{Data collection}

Data will be collected at the university facility at screening, baseline and post-intervention period (12 weeks after the start of the intervention) (Fig. 1). After providing baseline eligibility screening, potential participants will be offered a total of five visits for health assessments at the university facility. Participants in the intervention group will have an additional sixth visit for attending the mid-point focus group. All digital data (transcripts, digital information, health assessment databases) will be stored securely on the password-protected university computers available only to the research team. All other data (e.g. eligibility screening forms, activity data and questionnaire data) will be pseudo-anonymised with a unique ID number and stored in locked filing cabinets/ on password-protected university computers accessible only to the research team. Only the researchers will have access to the data.

\section{Sample size}

As this will be the feasibility study to inform the design of the future large-scale RCT, a total target sample of 40 older adult participants will be recruited for estimation of the critical parameters [63] with 20 in the intervention group and 20 in the wait-list control group.

\section{Recruitment}

Participants will be recruited from local neighbourhoods (households) and communities in Birmingham via a two-step strategy. First, through widespread advertisement 
in local media resources (newspaper, University of Birmingham website), via posters (Additional file 7) and distribution of leaflets about the study in local community centres and shops, churches, temples and mosques, local libraries, post offices, veterinary surgeries; and through advertisements via the University of Birmingham's 1000 Elders Group [64], and Ageing Better Consortium BVSC [65]. This is to recruit an ethnically diverse sample that is reflective of the Birmingham population. Secondly, recruitment will be facilitated during the eligibility screening where potential participants will be given a copy of the information sheet (Additional file 8) or a leaflet about the study and will be asked to invite anyone else they know and who might be interested in the project, i.e. using snowball or chain recruitment by word of mouth [66].

\section{Randomisation and concealment}

Randomisation will be conducted after completion of baseline measures using a computer-generated random sequence performed by an external researcher not involved in the delivery of the intervention or outcome assessment. Participants will be informed about the group allocation by e-mail or a phone call by a person not involved in assessments or delivery of the intervention. At the outcome assessment level, participants who will be assessors of their own psychosocial outcomes using questionnaires will be blinded to their group allocation at the time of completing the initial questionnaires. Intervention providers who will be responsible for outcome assessments will not be blinded to the intervention delivery as this would not be possible, given that the study and walks will be conducted by the $\mathrm{PhD}$ student (AS) who is the researcher.

\section{Recruitment and retention rates}

Recruitment will be aimed to be at a rate of 10 participants a month (to a minimum of 40 participants) for estimation of the critical parameters of the feasibility study [63]. If the number of recruited participants is less than $75 \%$ by the end of the 4-month recruitment period or if the retention rate is less than $75 \%$ at 12 weeks (end-point period), changes will be made to the recruitment strategy and the intervention will run again a few months later. No targets were set for other feasibility outcomes, e.g. questionnaire completion rates or attendance at the intervention sessions.

\section{Progression criteria}

As suggested by El-Kotob et al. [67], feasibility studies are not adequately powered to test the hypothesis of the efficacy of physical activity intervention, and therefore, a priori criteria for progression to the definitive large-scale RCT is advisable to consider future efforts. The progression criteria to a definitive large-scale RCT were (1) no any serious adverse events, such as hospitalisation, life-threatening condition, death and any adverse events associated with the intervention experienced by less than 5\% of participants per group; (2) recruitment rate of no less than $75 \%$ by the end of the 4-month recruitment period; and (3) retention rate of no less than $75 \%$ in each group at 12 weeks (end-point). If all the three criteria were not met, there would be insufficient evidence to justify proceeding to the definitive RCT. Changes will be required to be made to the intervention with the consequent re-running of the intervention a few months later.

\section{Statistical methods}

\section{Quantitative data analysis}

Quantitative data will be analysed using SPSS version 22.0 for Windows (SPSS Inc., Chicago, IL) employing an intention-to-treat analysis (based on their treatment allocation and irrespective of participants' adherence or withdrawal) [68]. The level of significance will be set at $p<.05$; however, any hypothesis testing is preliminary and results will be interpreted with caution as this pilot study is underpowered and the analyses based on small numbers. Baseline differences between groups for continuous data (e.g. age, BMI, resting blood pressure, number of comorbidities, cognitive and physical functioning, and outcomes of questionnaires) will be analysed using one-way ANOVA. Chi-squared tests will be applied for nominal data (e.g. gender, ethnicity, marital status, living arrangements, level of education, children and employment status). For descriptive statistics, data will be presented as means (M) and standard deviations (SD). Nominal data will be presented as number $(N)$ and percentage. Mixed between (group) and within (time) repeated-measures ANOVAs with post-hoc comparisons will be applied to investigate the effect of the intervention versus control on psychosocial outcomes (loneliness, social support, support networks, depression, anxiety, self-efficacy for exercise, satisfaction with level of social contacts), expected outcomes and barriers for exercise, and accelerometer data. The accelerometer data will be analysed using the ActivPAL software V7.1.18 (PAL technologies, Scotland, UK). Recorded data will be downloaded to a computer, and data for average daily amount of stepping (step counts), average time lying and sitting (h) in increments of $15 \mathrm{~s}$, average time standing $(\mathrm{h})$ and energy expenditure (EE, $\mathrm{MET} / \mathrm{h}$ ) will be analysed using mixed between (intervention group) and within (time) ANOVAs. For the Expected Outcomes and Barriers for Habitual Exercise scale [58], additional test-retest reliability will be calculated via correlation. In order to explore which outcome measures are likely to be the most important for the main trial, Pearson's correlations will be performed 
between calculated change scores over time in the experimental group for all psychosocial outcomes (Lubben's social networks, loneliness and self-efficacy for exercise) and change scores for averaged daily physical activity (time lying/sitting (h), time standing (h), time stepping (h), step counts, sit-to-stand transitions (n) and energy equivalent (METs/h)). A power calculation and sample size estimation for a future large-scale RCT will be calculated for meaningful outcomes (e.g. loneliness or social support) using the method based on the differences in means between the intervention and control group using the G-power software Version 3.1 [62].

\section{Qualitative data analysis}

Qualitative data will be thematically analysed by two research team members independently using a phenomenological inductive approach [61]. Transcripts will be returned to participants for comments/correction to ensure transparency and trustworthiness of the data (member checking) [69]. Validated transcripts will be read several times by two independent researchers to obtain an overall meaning. Then, themes and subthemes with important meanings common for all participants will be derived from the obtained data. Results will be compared through discussion between reviewers [70].

\section{Data storage and protection}

Research data will be kept for 10 years in line with UK data protection regulations [71]. Physical data will be pseudo-anonymised with a unique ID number and stored confidentially in locked filing cabinets/on password-protected university computers accessible only to the research team. Digitally recorded interview transcripts will be stored securely on a password-protected computer that only the researchers will have access to. Audio recordings will be destroyed after the recordings are transcribed verbatim.

\section{Discussion}

This study will explore the feasibility and acceptability of the Physical Activity Intervention for Loneliness (PAIL) in community-dwelling older adults. The key features of the study are based on effective components of physical activity interventions to treat loneliness obtained from a systematic review and meta-analysis of the existing evidence that highlighted the lack of physical activity interventions for older adults residing in community settings [23]. Studies to date either included non-PA interventions only or mixed design studies for only one or two outcomes (e.g. RCTs, case-control studies, longitudinal). The randomised controlled design of this feasibility study allows improvement upon the methodological rigour of the current evidence.
Consequently, this feasibility study uses objective measures of PA and comprehensive methods of assessment of social health which will allow estimation of the mechanisms of association between PA and social health outcomes in older adults in the future large-scale study. Findings from this study will be of the interest to healthcare professionals and community organisations working with older adults at risk of loneliness or social isolation in the implementation of exercise interventions to address this problem in society.

\section{Dissemination}

Dissemination will include a summary of the results for participants, publication of an open-access article detailing the full findings (also part of the PhD student's thesis) and presentation of study findings at conferences. The results of this feasibility study will be used to inform the development of a large-scale RCT. Participants in the intervention will be sent a feedback information sheet with the results of the study by e-mail or post (if no e-mail is available). The findings are also intended to be disseminated at the British Geriatric Society Loneliness annual meetings and via news at www.campaigntoendloneliness.org. The published study protocol and journal paper will be uploaded to the Current Research Information System (CRIS) - PURE portal available at pure.bham.ac.uk open to the academic staff, students and external researchers.

\section{Trial status}

This feasibility trial is registered at Clinicaltrials.gov Identifier: NCT03458793. Ethical approval for the project was received from the STEM Research Ethics Committee of the University of Birmingham, UK (ERN_16-1419A). Participant recruitment and baseline data collection started in October 2017 and is expected to be finished by November 2018. The study will be fully completed by May 2019 .

\section{Additional files}

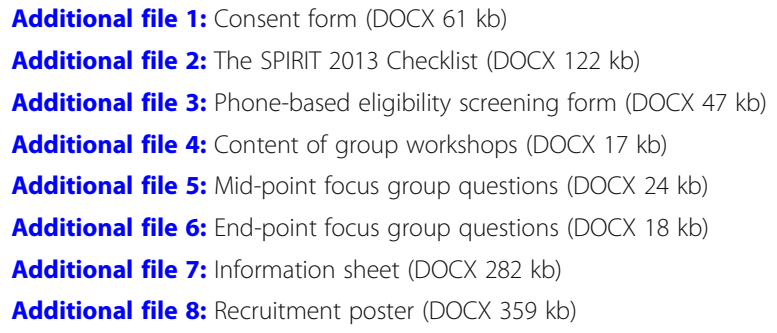

\section{Abbreviations}

ANOVA: Repeated-measures analysis of variance; BMI: Body mass index; BP rest: Resting blood pressure; BVSC: Birmingham Voluntary Service Council; EE: Energy expenditure; ES: Effect size; GP: General practitioner;

HADS: Hospital Anxiety and Depression Scale; HR max. Heart rate maximum; 
LSNS-6: 6-Item Lubben's Social Network Scale; M: Means; MET: Metabolic equivalent; MOCA: Montreal Cognitive Assessment; MOSSSS: Medical Outcomes Study Social Support Survey; N: Number; PA: Physical activity; PAIL: Physical Activity Intervention for Loneliness; RCT: Randomised controlled trial; RPE: Borg's Ratings of Perceived Exertions; SD: Standard deviations; SEE: Self-efficacy for exercise scale; SPPB: Short Physical Performance Battery: STEM Ethical Review Committee: Science, Technology, Engineering and Mathematics Ethical Review Committee; UCLA-8: 8-Item The University of California, Los Angeles Loneliness Scale; WLC group: Wait-list control group

\section{Acknowledgements}

The lead author of this review is funded by a Russian government-sponsored scholarship programme "Global Education", Moscow School of Management "Skolkovo" (Grant number 35). We would like to acknowledge Farhan Noordali (Doctoral Researcher, University of Birmingham) for acting as a moderator of the focus group interviews and Meike Rosemeyer (PhD student, University of Birmingham) for helping to assist with focus group interviews.

\section{Disclosure statement}

The authors of this review declare that they have no known conflicts of interest.

\section{Funding}

The lead author of this paper is funded by a Russian government-sponsored scholarship programme "Global Education", Moscow School of Management "Skolkovo" (Grant number 35).

\section{Availability of data and materials}

Not applicable.

\section{Authors' contributions}

AW, JT and CG helped in the development of trial design and study protocol, assisted in obtaining ethics approval and amendments and helped in facilitating patient recruitment, register study protocol and structure the research stages and overseeing all study procedures. JT also helped to design the focus group interviews. The doctoral research student AS obtained funding and was involved in the design of the study protocol, participant recruitment, health assessments and implementation of the intervention.

\section{Authors' information}

AW is a Professor in Behavioural Medicine. JT is a Professor of Public Health Nutrition and Exercise. CG is a Reader in Musculoskeletal Ageing and Health, all at the School of Sport, Exercise \& Rehabilitation Sciences, University of Birmingham, UK.

\section{Consent for publication}

Not applicable.

\section{Competing interests}

The authors declare that they have no competing interests.

\section{Publisher's Note}

Springer Nature remains neutral with regard to jurisdictional claims in published maps and institutional affiliations.

\section{Author details}

${ }^{1}$ School of Sport, Exercise and Rehabilitation Sciences, University of Birmingham, Birmingham, UK. ${ }^{2}$ MRC - Arthritis Research UK Centre for Musculoskeletal Ageing Research, University of Birmingham, Birmingham, UK.

Received: 29 June 2018 Accepted: 29 November 2018

\section{Published online: 19 December 2018}

\section{References}

1. Cacioppo JT, Cacioppo S. Social relationships and health: the toxic effects of perceived social isolation. Soc Personal Psychol Compass. 2014;8:58-72. https://doi.org/10.1111/spc3.12087.

2. Masi CM, Chen H-Y, Hawkley LC, et al. A meta-analysis of interventions to reduce loneliness. Personal Soc Psychol Rev. 2010:219-66.

3. Netz Y, Wu M-J, Becker BJ, et al. Physical activity and psychological wellbeing in advanced age: a meta-analysis of intervention studies. Psychol Aging. 2005;20:272-84.
4. Office for National Statistics. Inequalities in social capital by age and sex July 20152015 [http://webarchive.nationalarchives.gov.uk/20160105160709/ http://www.ons.gov.uk/ons/rel/wellbeing/measuring-national-well-being/ inequalities-in-social-capital-by-age-and-sex/art.html].

5. Peplau LA, Cutrona CE. The revised UCLA Loneliness Scale: concurrent and discriminant validity evidence. J Pers Soc Psychol. 1980;39:472-80.

6. Holt-Lunstad J, Smith TB, Layton JB. Social relationships and mortality risk: a meta-analytic review. PLoS Med. 2010;7:e1000316.

7. Steptoe A, Shankar A, Demakakos P, et al. Social isolation, loneliness, and allcause mortality in older men and women. Proc Natl Acad Sci. 2013;110: 5797-801.

8. Age UK. Later life in the United Kingdom 2017 [https://www.ageuk.org.uk/ Documents/EN-GB/Factsheets/Later_Life_UK_factsheet.pdf?dtrk=true].

9. World Health Organisation. Active ageing: a policy framework. Geneva: 2002.

10. Bernard SM, Perry H. Loneliness and social isolation among older people in North Yorkshire: stage 2 report; 2013.

11. Jopling K. Promising approaches to reducing loneliness and isolation in later life. London: Age UK; 2015. http://www.ageuk.org.uk/Documents/ENGB/For-professionals/Policy/Promising_approaches-loneliness_and_isolation. pdf?dtrk=true

12. Milligan C, Dowrick C, Payne S, et al. Men's sheds and other gendered interventions for older men: improving health and wellbeing through social activity-a systematic review and scoping of the evidence base. Lancaster University Centre for Ageing Research: Lancaster; 2013.

13. American College of Sports Medicine. ACSM's guidelines for exercise testing and prescription: Lippincott Williams \& Wilkins; 2013.

14. Caspersen CJ, Powell KE, Christenson GM. Physical activity, exercise, and physical fitness: definitions and distinctions for health-related research. Public Health Rep. 1985;100:126.

15. Pels F, Kleinert J. Loneliness and physical activity: a systematic review. International Review of Sport and Exercise Psychology. 2016;9:1-30. https:// doi.org/10.1080/1750984X.2016.1177849.

16. Biddle SJ, Asare M. Physical activity and mental health in children and adolescents: a review of reviews. Br J Sports Med. 2011:45:886-96.

17. Stevens N. Combating loneliness: a friendship enrichment programme for older women. Ageing and Society. 2001;21:183-202. https://doi.org/10.1017/ S0144686X01008108

18. Ferraro KF, Farmer MM. Social compensation in adulthood and later life Compensating for Psychological Deficits and Declines: Managing Losses and Promoting Gain. 1995:127-45.

19. Fredrickson BL. The broaden-and-build theory of positive emotions. Philosophical Transactions-Royal Society of London Series B Biological Sciences. 2004:1367-78. https://doi.org/10.1098/rstb.2004.1512.

20. Newall NE, Chipperfield JG, Bailis DS, et al. Consequences of loneliness on physical activity and mortality in older adults and the power of positive emotions. Health Psychology. 2013;32:921. https://doi.org/10.1037/a0029413.

21. Henry KB, Arrow H, Carini B. A tripartite model of group identification theory and measurement. Small Group Res. 1999;30:558-81.

22. Cohen S, Wills TA. Stress, social support, and the buffering hypothesis, Psychol Bull. 1985;98:310.

23. Shvedko A, Whittaker AC, Thompson JL, et al. Physical activity interventions for treatment of social isolation, loneliness or low social support in older adults: a systematic review and meta-analysis of randomised controlled trials. Psychology of Sport and Exercise. 2018;34:128-37. https://doi.org/10. 1016/.jpsychsport.2017.10.003.

24. Cattan M, White $\mathrm{M}$, Bond J, et al. Preventing social isolation and loneliness among older people: a systematic review of health promotion interventions. Ageing Soc. 2005;25:41-67.

25. Cohen-Mansfield J, Hazan H, Lerman Y, et al. Correlates and predictors of loneliness in older-adults: a review of quantitative results informed by qualitative insights. Int Psychogeriatr. 2016;28:557-76.

26. Dickens AP, Richards SH, Greaves $\mathrm{C}$, et al. Interventions targeting social isolation in older people: a systematic review. BMC Public Health. 2011;11:1-22.

27. Hagan R, Manktelow R, Taylor BJ, et al. Reducing loneliness amongst older people: a systematic search and narrative review. Aging Ment Health. 2014;18:683-93.

28. Petitte T, Mallow J, Barnes E, et al. A systematic review of loneliness and common chronic physical conditions in adults. The open psychology journal. 2015;8:113-32.

29. Smith GL, Banting L, Eime R, et al. The association between social support and physical activity in older adults: a systematic review. Int J Behav Nutr Phys Act. 2017;14:56. https://doi.org/10.1186/s12966-017-0509-8. 
30. Snowden MB, Steinman LE, Carlson WL, et al. Effect of physical activity, social support, and skills training on late-life emotional health: a systematic literature review and implications for public health research. Front Public Health. 2014:2:1-14.

31. Toepoel V. Ageing, leisure, and social connectedness: how could leisure help reduce social isolation of older people? Soc Indic Res. 2013;113:355-72.

32. Bandura A. Perceived self-efficacy in cognitive development and functioning. Educ Psychol. 1993;28:117-48.

33. Fry PS. Predictors of health-related quality of life perspectives, self-esteem, and life satisfactions of older adults following spousal loss an 18-month follow-up study of widows and widowers. The Gerontologist. 2001;41:787-98.

34. Fry PS, Debats DL. Self-efficacy beliefs as predictors of loneliness and psychological distress in older adults. Int J Aging Hum Dev. 2002;55:233-69.

35. McAuley E, Blissmer B. Social relations, physical activity, and well-being in older adults. Prev Med. 2000;31:608-17.

36. Taliaferro LA, Rienzo BA, Miller DM, et al. Potential mediating pathways through which sports participation relates to reduced risk of suicidal ideation. Res Q Exerc Sport. 2010;81:328-39.

37. Holahan CK, Holahan CJ. Self-efficacy, social support, and depression in aging: a longitudinal analysis. J Gerontol. 1987;42:65-8.

38. Stevens M, Hillsdon M, Thorogood M, et al. Cost-effectiveness of a primary care based physical activity intervention in 45-74 year old men and women: a randomised controlled trial. Br J Sports Med. 1998;32:236-41.

39. Hughes ME, Waite $L$, Hawkley LC, et al. A short scale for measuring loneliness in large surveys results from two population-based studies. Research on aging. 2004;26:655-72

40. Guralnik JM, Simonsick EM, Ferrucci L, et al. A short physical performance battery assessing lower extremity function: association with self-reported disability and prediction of mortality and nursing home admission. Journal of Gerontology: Medical Sciences. 1994;49:M85-94.

41. Pahor M, Guralnik JM, Ambrosius WT, et al. Effect of structured physical activity on prevention of major mobility disability in older adults: the LIFE study randomized clinical trial. JAMA. 2014;311:2387-96. https://doi.org/10. 1001/jama.2014.5616.

42. Nasreddine ZS, Phillips NA, Bédirian V, et al. The Montreal Cognitive Assessment, MoCA: a brief screening tool for mild cognitive impairment. JAGS. 2005;53:695-9. https://doi.org/10.1111/j.1532-5415.2005.53221.x.

43. Freitas $S$, Simões MR, Alves $L$, et al. Montreal cognitive assessment: validation study for mild cognitive impairment and Alzheimer disease. Alzheimer Dis Assoc Disord. 2013;27:37-43.

44. Shephard RJ. Readiness for physical activity: President's Council on Physical Fitness and Sports; 1994

45. Long JE, Ring C, Drayson $M$, et al. Vaccination response following aerobic exercise: can a brisk walk enhance antibody response to pneumococcal and influenza vaccinations? Brain Behav Immun. 2012;26:680-7. https://doi.org/ 10.1016/j.bbi.2012.02.004

46. Fox SM, Naughton JP, Haskell WL. Physical activity and the prevention of coronary heart disease. Ann Clin Res. 1971;3:404-32.

47. Borg E, Kaijser L. A comparison between three rating scales for perceived exertion and two different work tests. Scand J Med Sci Sports. 2006;16:57-69.

48. Persinger R, Foster C, Gibson M, et al. Consistency of the talk test for exercise prescription. Med Sci Sports and Exerc. 2004;36:1632-6. https://doi. org/10.1249/01.MSS.0000074670.03001.98

49. Borg G. Borg's perceived exertion and pain scales: human kinetics; 1998.

50. Department of Health. UK Physical Activity Guidelines 2011 [https://www. gov.uk/government/publications/uk-physical-activity-guidelines].

51. Chastin S, Granat M. Methods for objective measure, quantification and analysis of sedentary behaviour and inactivity. Gait \& Posture. 2010;31:82-86. doi: https://doi.org/10.1016/j.gaitpost.2009.09.002.

52. Hays RD, DiMatteo MR. A short-form measure of loneliness. J Pers Assess. 1987:51:69-81

53. Sherbourne CD, Stewart AL. The MOS social support survey. Soc Sci Med. 1991;32:705-14.

54. Lubben J, Blozik E, Gillmann G, et al. Performance of an abbreviated version of the Lubben Social Network Scale among three European community-dwelling older adult populations. The Gerontologist. 2006; 46:503-13.

55. Zigmond AS, Snaith RP. The hospital anxiety and depression scale. Acta Psychiatr Scand. 1983;67:361-70.

56. Resnick $B$, Jenkins LS. Testing the reliability and validity of the self-efficacy for exercise scale. Nurs Res. 2000;49:154-9.
57. Fokkema T, Knipscheer K. Escape loneliness by going digital: a quantitative and qualitative evaluation of a Dutch experiment in using ECT to overcome loneliness among older adults. Ageing \& Mental Health. 2007;11:496-504. https://doi.org/10.1080/13607860701366129.

58. Steinhardt MA, Dishman RK. Reliability and validity of expected outcomes and barriers for habitual physical activity. J Occup Environ Med. 1989;31:536-46.

59. Tong A, Sainsbury P, Craig J. Consolidated criteria for reporting qualitative research (COREQ): a 32-item checklist for interviews and focus groups. Int J Qual Health Care. 2007;19:349-57.

60. MacDougall C, Fudge E. Planning and recruiting the sample for focus groups and in-depth interviews. Qual Health Res. 2001;11:117-26.

61. Creswell JW. Qualitative inquiry and research design: choosing among five approaches: sage publications; 2012.

62. Erdfelder E, Faul F, Buchner A. GPOWER: a general power analysis program. Behav Res Methods Instrum Comput. 1996;28:1-11.

63. Arain M, Campbell MJ, Cooper CL, et al. What is a pilot or feasibility study? A review of current practice and editorial policy. BMC Medical Research Methodology. 2010;10:67.

64. The Birmingham 1000 Elders Group. 2017 [https://www.birmingham.ac.uk/ research/activity/mds/centres/healthy-ageing/elders.aspx].

65. Birmingham Voluntary Service Council. The consortium of ageing better 2016 [https://www.bvsc.org/].

66. Miles MB, Huberman AM. Qualitative data analysis: a sourcebook. Beverly Hills: Sage Publications; 1994.

67. El-Kotob R, Giangregorio LM. Pilot and feasibility studies in exercise, physical activity, or rehabilitation research. Pilot and feasibility studies. 2018:4(1):137.

68. Gupta SK. Intention-to-treat concept: a review. Perspectives in Clinical Research. 2011;2:109-12. https://doi.org/10.4103/2229-3485.83221.

69. Fossey E, Harvey C, McDermott F, et al. Understanding and evaluating qualitative research. Aust N Z J Psychiatry. 2002;36:717-32.

70. Tesch R. Qualitative research: analysis types and software: Routledge; 2013.

71. Carey P. Data protection: a practical guide to UK and EU law: Oxford University Press, Inc.; 2018
Ready to submit your research? Choose BMC and benefit from:

- fast, convenient online submission

- thorough peer review by experienced researchers in your field

- rapid publication on acceptance

- support for research data, including large and complex data types

- gold Open Access which fosters wider collaboration and increased citations

- maximum visibility for your research: over $100 \mathrm{M}$ website views per year

At $\mathrm{BMC}$, research is always in progress.

Learn more biomedcentral.com/submissions 